\title{
Environment maps and the perception of shape from mirror reflections
}

\author{
Arthur Faisman* \\ School of Computer Science \\ McGill University
}

\author{
Michael Langer ${ }^{\dagger}$ \\ School of Computer Science \\ McGill University
}
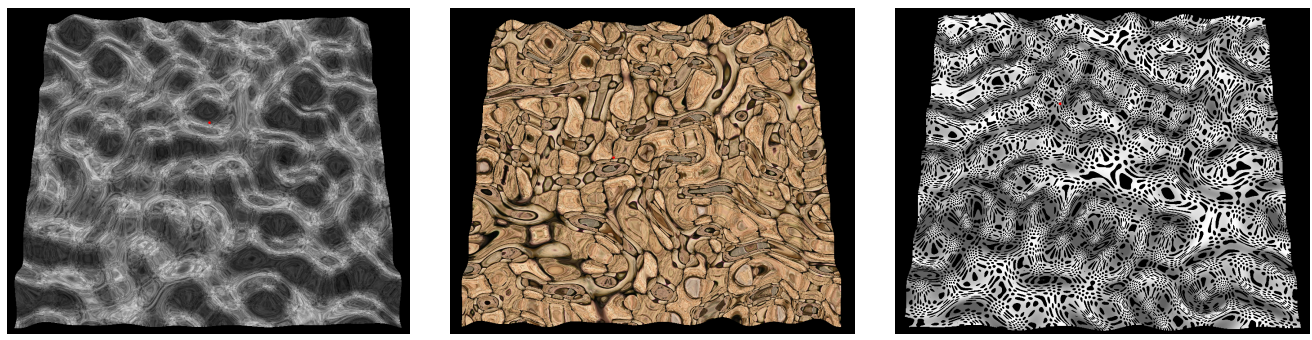

Figure 1: Three example images are shown: (a) 1/f texture modulated by a light envelope that peaks at the zenith, (b) a texture used in our previous study, and (c) a soccer ball texture modulated by an envelope that peaks in the direction of the surface normal.

CR Categories: I.3.7 [Computer Graphics]: Three-Dimensional Graphics and Realism-Color, shading, shadowing, and texture;

Keywords: shape, perception, environment maps, mirror, lighting

\section{Introduction}

Environment maps are commonly used in computer graphics to approximate the appearance of smoothly curved surfaces with mirrorlike reflectance. Perceiving the shape of such surfaces is a challenging task for the visual system, however, because the image intensities are determined both by the surface shape and by the environment [Fleming et al. 2004]. Here we extend our recent study of the perception of qualitative shape of mirror surfaces [Faisman and Langer 2013] by examining more closely how qualitative shape perception varies with the environment maps that are used.

\section{Method}

We rendered smooth, bumpy mirror terrain surfaces using OpenGL environment mapping. Images were presented to naive observers under monocular viewing, and observers were asked to judge whether marked points were on a hill or in a valley.

Several different environment maps were used in the renderings including the textures used in our previous study as well as three new ones: $1 / f$ noise, a near regular soccer ball pattern, and a constant intensity (white). For each of these basic environment map textures, we included conditions in which each texture was multiplied by a low frequency envelope to give it a dominant direction. The envelope itself produced a low frequency shading-like effect similar to soft gloss. The peak of this multiplicative envelope was either at the zenith as in [Faisman and Langer 2013], or in the direction of the overall surface normal. In our previous study, using a more limited set of textures, we showed that performance was higher when the zenith envelope was used than when no envelope was used. But it was unclear if this performance difference was due to the presence of a low frequency shading cue per se or if it was due to the specific envelope used. Including the condition with a modulation

*e-mail:arthur.faisman@mail.mcgill.ca

†e-mail:langer@cim.mcgill.ca peaking in the direction of the surface normal allowed us to address this question.

\section{Results and Conclusions}

As shown in the plots below, performance was highest when the envelope peaked at the zenith, lowest when the envelope peaked at the direction of the surface normal, and in between when no envelope was used. We also found that performance seemed to systematically differ between the underlying textures used, e.g. $1 / f$ texture gave better performance than the soccer ball texture.

We conclude that shape perception of mirror surfaces depends signficantly on the environment map that is used. Moreover, since natural environments often contain visual structures at many scales $(1 / f$ noise) and are dominated by an overall light from above, such environments can provide rich cues for shape perception.

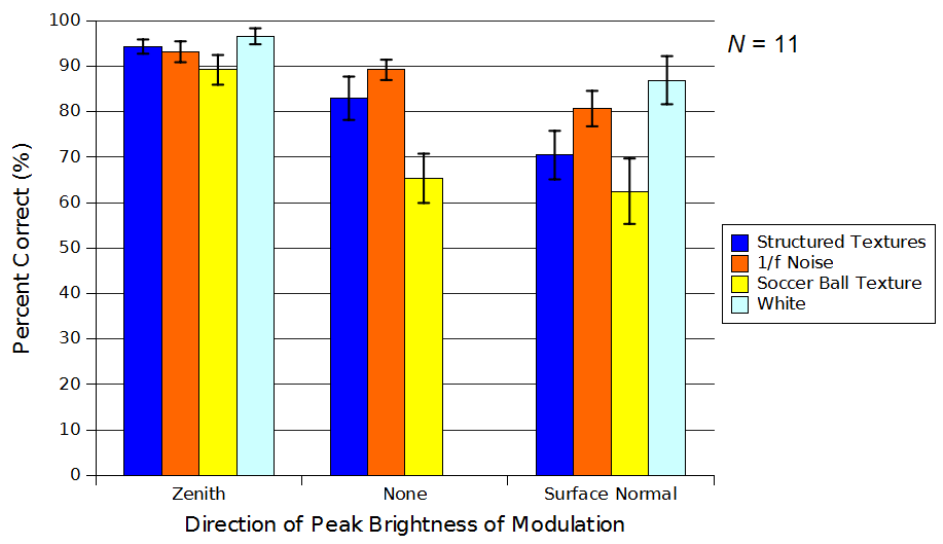

\section{References}

FAisman, A., AND LANGER, M. S. 2013. Qualitative shape from shading, highlights, and mirror reflections. Journal of Vision 13, 5 .

Fleming, R. W., Torralba, A., And Adelson, E. H. 2004. Specular reflections and the perception of shape. Journal of $\mathrm{Vi}$ sion 4, 798-820. 